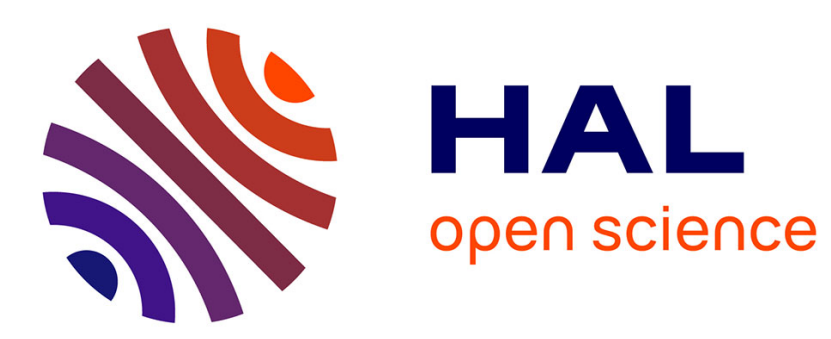

\title{
Interstrand Void Content evolution in compression moulding of Randomly Oriented Strands (ROS) of thermoplastic composites
}

\author{
Arthur Lévy, Pascal Hubert
}

\section{- To cite this version:}

Arthur Lévy, Pascal Hubert. Interstrand Void Content evolution in compression moulding of Randomly Oriented Strands (ROS) of thermoplastic composites. Composites Part A: Applied Science and Manufacturing, 2015, 70, pp.121-131. hal-03191079

\section{HAL Id: hal-03191079 \\ https://hal.science/hal-03191079}

Submitted on 6 Apr 2021

HAL is a multi-disciplinary open access archive for the deposit and dissemination of scientific research documents, whether they are published or not. The documents may come from teaching and research institutions in France or abroad, or from public or private research centers.
L'archive ouverte pluridisciplinaire HAL, est destinée au dépôt et à la diffusion de documents scientifiques de niveau recherche, publiés ou non, émanant des établissements d'enseignement et de recherche français ou étrangers, des laboratoires publics ou privés. 


\title{
Interstrand Void Content evolution in compression moulding of Randomly Oriented Strands (ROS) of thermoplastic composites
}

\author{
Arthur Levy, Pascal Hubert* \\ Department of Mechanical Engineering, McGill University, 817 Sherbrooke St. West, Montréal QC, H3A 0C3, Canada.
}

\begin{abstract}
Compression moulding of randomly oriented strands (ROS) of thermoplastic composite is a process that enables the forming of complex shapes with keeping final properties close to that of continuous fibre composites. During forming several deformation mechanisms occur. In this paper we focus on the interstrand void content (ISVC) reduction: the squeezing of each single strand during compression enables filling of the gaps between strands. A modelling of this deformation mechanism was developed. The compaction is ruled by an ordinary differential equation that was solved numerically. The model was validated experimentally using an instrumented hot press with Carbon-PEEK prepreg strands. The model accurately predicted ISVC in four characteristic cases. Using the proposed model, the influence of several process and material parameters were investigated. Finally, a design chart giving the final ISVC for a wide range of pressure, strand geometry and part thickness, was constructed.
\end{abstract}

Keywords: A. Discontinuous reinforcement, A. Thermoplastic resin, B. Porosity, E. Compression moulding

\section{Nomenclature}

$\delta \quad$ initial strand thickness

$\delta^{380} \quad$ initial strand thickness at processing temperature

$\{\boldsymbol{X}\} \quad$ unknown, concatenation of $h$ and $a_{i}$

$\mu \quad$ equivalent viscosity of the melt

$\phi \quad$ interstrand void content

$\rho \quad$ material density

\footnotetext{
* Corresponding author, +1 5143986303

Email addresses: arthur.levy@univ-nantes.fr (Arthur Levy), pascal.hubert@mcgill.ca (Pascal Hubert)
} 
$\tilde{n} \quad$ mean number of strands in the thickness

A total area of the mould

$a_{i} \quad$ area covered by $i$ strands

$a_{i, 0} \quad$ initial area covered with $i$ strands

F $\quad$ closing force applied by the press

$F_{1} \quad$ closing force applied on several adjacent strands

$F_{k} \quad$ closing force applied on a stack of strands

$F_{\text {one }} \quad$ closing force applied on one single strand

$h \quad$ gap between platens

$h_{f} \quad$ final gap between platens

$h_{f}^{380} \quad$ final gap between platens at processing temperature

$h_{\infty} \quad$ final nominal thickness of the specimen

$h_{n d} \quad$ non deformable height

$j \quad$ index of the smallest columns being currently squeezed

$L_{0} \quad$ initial strand length

$n \quad$ number of strands in the highest stack

$N_{s} \quad$ total number of strands in the mould

$P \quad$ closing pressure $F / A$

$p \quad$ number of strands in the lowest column

$s \quad$ interstrand stacking number

$t \quad$ time

$V_{m} \quad$ volume of matter

$w \quad$ strand width

$w_{0} \quad$ initial strand width 


\section{Introduction}

Continuous fibres (CF) thermoplastic composites, while exhibiting good mechanical performance, are difficult to form. Forming processes for $\mathrm{CF}$ (such as automatic prepreg tape placement or stamping) are limited to simple geometries with minimal curvature and thickness variations. On the other hand, automotive industry can produce parts with intricate features made from flow moulding compounds $[1,2]$, or short fibre reinforced injection moulded thermoplastic, but their mechanical properties are generally too low for aerospace applications.

Randomly Oriented Strands (ROS)

Lying between these two extremes, randomly oriented strands of unidirectional prepreg composite tape (ROS), with high fibre volume fractions (> 50\%), have attractive forming and mechanical characteristics [36]. This paper focuses on the processing of ROS thermoplastic composites by compression moulding.

In this process strands of thermoplastic composite tapes are positioned randomly in a mould cavity (close-up in Fig. 7a). The setup is then compressed under a hot press where a force is applied to consolidate the material. Complex shapes including corners, thickness changes, ribs or holes can be obtained in one compression moulding step [7].

The deformation that the material undergoes during press forming of ROS determines the feasibility of the process, and the final quality of the part. The deformation mechanisms occurring during press forming of ROS are rather similar to those encountered in randomly distributed fibre bundles such as sheet moulding compound forming. Picher-Martel et al. [6] assumed that during compression moulding of ROS, the material undergoes three different deformation modes:

1. An elastic compaction behaviour, denoted as packing deformation, at low load. It is mostly associated to the bending and conformation of the strands. Luchoo et al. [8] proposed a simulation of this elastic response at the scale of the strand. Servais et al. [9] suggested an experimental procedure to determine the packing stress in the case of fibre bundle suspension. Their experiments conducted on isolated fibre bundles showed that the bundles deformed at packing stresses lower than the stresses involved in the material forming. Hereunder, following the same idea, the normal stresses involved while press forming ROS is considered way higher than the packing stress. The packing deformation therefore occurs only at very low load during the initial stage of the process.

2. A macroscopic squeeze flow at the scale of the part. At this scale the strands melt undergoes a squeeze flow imposed by the hot press. Considering the strands melt as an homogeneous medium, its viscous behaviour is intimately linked to the sliding between strands $[6,10]$. The macroscopic squeeze flow ensures that material will flow and fill intricate features. These areas, such as corners or ribs, may indeed have remained empty after the initial operator positioning of the strands in the mould. 
3. A flow at the mesoscopic scale of the strand. In this mode, the applied pressure results in a spreading of each individual strand. With this squeezing, the gaps between strands reduce. This mechanism is denoted as the interstrand void content reduction. These macroscopic interstrand voids have to reduce enough to prevent dramatic property loss in the final part. It is a usual rule of thumb in aeronautical industry to guarantee a void content below $1 \%[11,12]$.

The present study aims at modelling and predicting this latter phenomenon, in order to better understand its sensitivity to the process parameters (pressure, strand geometry, temperature...). Note that beside the macroscopic interstrand voids, the prepreg strands also contain microscopic intrastrand voids. This microscopic void collapse, that also occur during consolidation, is a different mechanism. It has been studied in the past for other manufacturing processes $[13,14]$. The microscopic intrastrand voids reduction is not addressed in this paper. Thus, fully consolidated individual strands are considered.

The difficulty of the very large number of strands and their random configurations is addressed in section 2 , where the column representation is introduced. Then, in section 3 working in this column representation, the squeeze flow behaviour of a single strand is depicted, and extended to several non overlapping strands, stacks of strand, and ultimately the general ROS case. A global behaviour for ROS thickness and interstrand void content (ISVC) evolution is obtained. Finally a solving scheme is proposed to numerically solve for the obtained model.

In section 4, the Carbon/PEEK thermoplastic composite material used in the study is first presented. The experimental setup consisting of an instrumented hot press is then described. The platen gap is measured in situ while press forming ROS samples at a controlled temperature. Finally, experimental measurements validate the proposed model.

In the last section, the presented model is used to investigate the effect of various process parameters on the final ISVC. Ultimately a guideline chart is constructed to help determining the adequate processing parameters for ROS press forming that ensure a good final quality in terms of ISVC.

\section{Column representation}

The behaviour of each strands at their mesoscopic scale is needed to predict the ISVC evolution. Nonetheless, because of the very high number of strands in the mould (several thousands), studying the deformation of each strand on the real geometry is not conceivable. On the contrary, original approaches should describe the random configuration of strands in a simpler way. With this aim, studying the forming of random wood flakes panels, Dai and Steiner [15] adopted the column representation. The random configuration is described using a series of characteristic areas $a_{i}$. Based on statistical work [16], the series is known for typical positioning of strands in the mould, amongst which random positioning. In the present work, the column representation is adopted to describe the strand positioning in the mould. 
Following the approach by Dai and Steiner $[15,17]$, is of interest the number of strands in the thickness at each point of a plate. The random filling of the mould with strands consists in a particular realization. The total area $a_{i}(t)$ in the mould surface, containing $i$ strands in the thickness, is therefore a random variable. $a_{i}(t)$ is a function of time $t$. The distribution of strands in the mould can be represented graphically using the series of columns of different area $a_{i}(t)$, each with an initial thickness $\delta \times i, \delta$ being the initial thickness of one single strand.

\subsection{First example}

As a basic example of the column representation concept, figure 1a shows one realization consisting of the stacking of two overlapping strands of dimensions $L_{0} w_{0} \delta$. The column representation of such a stacking results in three columns, as shown on figure $1 \mathrm{~b}$ :

- one of thickness $2 \delta$ with an area $w_{0}^{2}$

- one of thickness $\delta$ with an area $2 w_{0}\left(L_{0}-w_{0}\right)$

- and one column of thickness 0 with an area $\left(L_{0}-w_{0}\right)^{2}$.

\subsection{Ideal random distribution}

In the general case of in-plane randomly oriented strands, the random variable $a_{i}$ has been shown to follow the Poisson distribution [16]:

$$
\frac{a_{i}}{A}=\frac{\tilde{n}^{i} \exp (-\tilde{n})}{i !}
$$

where $\tilde{n}$ is the mean number of strands through the thickness, and $A$ is the total area of the mould. $\tilde{n}$ is known and is the total strand area divided by the sample area $A[17]$ :

$$
\tilde{n}=\frac{N_{s} w_{0} L_{0}}{A}
$$

$N_{s}$ being the total number of strands. Note that alternatively, knowing the expected final nominal thickness one can deduce $\tilde{n}$ by:

$$
\tilde{n} \delta=h_{\infty}
$$

For the case where $\tilde{n}=20$, equation (1) gives a column representation such as the one shown in figure 2 .

\subsection{Realization of random distributions}

The Poisson distribution equation (1) is valid for a perfectly random distribution of the strands in the mould. In other words, the initial distribution of $a_{i}$ approaches the Poisson distribution when the number of strands increases. In the case of a finite number of strands in the mould the actual distribution $a_{i}$ will obviously deviate from this ideal Poisson distribution.

As given on equations (2) and (3), the number $N_{s}$ of strands in the mould depends on the geometry of the strands $w_{0}, L_{0}$ and $\delta$, the mould area $A$ and the expected final sample thickness $h_{\infty}$. Three cases shown on table 1 were investigated: 
- The reference case.

- The extreme case with very few strands is obtained with large strands geometry and a very small mould.

- The industrial case is a scale up of the reference case with a larger mould leading to a larger number of strands $N_{s}$.

For each case, a particular realization of the initial distribution $a_{i}$ can be generated using the algorithm proposed by Knuth [18, p. 137]. As shown in figure 3, the higher the number $N_{s}$ of strands, the closer $a_{i}$ is from the ideal Poisson distribution equation (1).

\section{ISVC modelling}

In this section, a modelling of the ISVC is developed using the column representation. First, the composite material is considered incompressible. The volume of matter, excluding interstrand voids,

$$
V_{m}=h_{\infty} A
$$

is therefore constant. During the compression, knowing the gap between platens $h(t)$, the volume of the sample including voids writes

$$
V(t)=h(t) A \text {. }
$$

Therefore the ISVC, $\phi$, is directly obtain from the platen gap $h$ as

$$
\phi(t)=\frac{V(t)-V_{m}}{V(t)}=\frac{h(t)-h_{\infty}}{h(t)} .
$$

The following sections will focus on predicting the gap evolution $h(t)$. Equation $(6)$ gives the ISVC evolution as a simple post-processing of $h(t)$.

\subsection{Constitutive compaction models}

In order to predict the behaviour of random wood flakes panels while compress formed, Dai and Steiner [17] propose to translate the elastic mechanical behaviour of one single flake to the column representation. They end up with a global behaviour representative of the randomly positioned flakes system. Similarly, in the present paper, the leading phenomenon is the deformation of the composite strands during hot press forming. But at processing temperature the thermoplastic matrix is molten. Therefore, unlike Dai and Steiner [17], the material undergoes finite non-elastic deformation. This leads to an evolution of the geometry that results in: (i) the closing of the interstrand voids and (ii) the evolution of the geometry in the column representation. In order to obtain the global behaviour of the ROS, the behaviour of one single strand has to be translated to the column representation first. 


\subsubsection{Squeeze flow of a single strand}

Each strand is subjected to a squeeze flow. Given the high number of fibres in the thickness of one strand (couple of tens), the material is considered as homogeneous at the mesoscopic scale of the strand.

As a first step the squeeze flow of a single strand such as the one shown in Figure $4 \mathrm{a}$ is considered. The strand of length $L_{0}$, width $w(t)$ and initial thickness $\delta$, is squeezed with a closing force $F_{\text {one }}$. Many authors studied such squeeze flow using Newtonian [19-21] or quasi-Newtonian behaviour [19, 20,22]. The strand being unidirectional, the fibres restrict the flow to a two dimensional plane strain squeeze flow (figure $4 \mathrm{~b}$ ). No slip boundary conditions are considered at the top and bottom interfaces, in contact with the hot plates. In this study, as a first assumption, the composite melt is supposed to behave as a Newtonian fluid with an equivalent viscosity $\mu$. Because $h(t) \ll w(t)$ lubrication assumption stands and leads to a strand thickness evolution $h(t)$ that obeys [19-21, 23]:

$$
\frac{d h}{d t}=-\frac{F_{\text {one }}}{L_{0} \mu} \frac{h(t)^{3}}{w(t)^{3}}
$$

The composite being incompressible, the current width of the strand $w(t)$ ensures:

$$
w(t) h(t)=w_{0} \delta
$$

such that

$$
\frac{d h}{d t}=-\frac{F_{\text {one }}}{L_{0} \mu} \frac{h(t)^{6}}{w_{0}^{3} \delta^{3}}
$$

or similarly

$$
F_{\text {one }}=-L_{0} \mu \frac{\partial h}{\partial t} \frac{w_{0}^{3} \delta^{3}}{h(t)^{6}} .
$$

Equation (10) shows that the closing force $F_{\text {one }}$ is inversely proportional to the sixth power of the current strand thickness $h(t)$. This high dependency is due to: (i) the widening of the strand while it is spread, making the reacting force higher because of a larger reacting surface and (ii) the thinning of the strand making the squeeze flow more difficult.

\subsubsection{Adjacent strands}

This single strand model is extended to the case of $i$ non overlapping strands. In the column representation, it results in one column of height $\delta$ with an area $a_{1}(t)$ and one empty column with an area $a_{0}(t)$. Even though there is one single column with strands, the strands are actually randomly positioned in the plate, as illustrated in figure 5a. Initially the area of the column 1 is:

$$
a_{1,0}=N_{s} w_{0} L_{0}
$$

where $N_{s}$ is the number of strands. Considering that the total closing force $F_{1}$ on the column is homogeneously split, the applied force $F_{s}$ on each strands writes:

$$
F_{s}=\frac{F_{1}}{i}=\frac{F_{1} w_{0} L_{0}}{a_{1,0}} .
$$


Each strand then deforms as dictated by equation (10), such that:

$$
\begin{aligned}
\frac{d h}{d t} & =-\frac{F_{s}}{L_{0} \mu} \frac{h^{6}}{w_{0}^{3} \delta^{3}} \\
& =-\frac{F_{1}}{\mu} \frac{h^{6}}{a_{1,0} w_{0}^{2} \delta^{3}}
\end{aligned},
$$

or similarly:

$$
F_{1}=-\mu \frac{d h}{d t} \frac{w_{0}^{2} \delta^{3}}{h^{6}} a_{1,0} .
$$

\subsubsection{A stack of strands}

For a stack of $k$ strands, the squeeze flow behaviour of each single strand is different. Previously, a zero velocity at the strand/mould boundary was considered because no slip occurred. In the present case the strand/strand interface can have a nonzero velocity. Two extreme cases are:

1. A unidirectional stacking of strands, where all the strands are stacked with the same orientation. In this case, it is similar to having one single thick strand (which thickness is the sum of the thicknesses of each stacked strand) as shown in figure 6a. Equation (14) is still valid substituting $\delta$ by $k \delta$ :

$$
F_{k}^{(1)}=-\mu \frac{d h}{d t} \frac{w_{0}^{2} \delta^{3}}{h^{6}} a_{k, 0} k^{3} .
$$

2. On the other hand, considering a $\left[0,90^{\circ}\right]$ stacking, the flow is restricted in the transverse direction at each strand interface. The squeeze flow kinematic is then similar, in each strand, to the case modelled previously (see figure $6 \mathrm{~b}$ ). Substituting $h$ with $h / k$ in equation (14) gives:

$$
F_{k}^{(2)}=-\mu \frac{d\left(\frac{h}{k}\right)}{d t} \frac{w_{0}^{2} \delta^{3}}{\left(\frac{h}{k}\right)^{6}} a_{k, 0}
$$

such that

$$
F_{k}^{(2)}=-\mu \frac{d h}{d t} \frac{w_{0}^{2} \delta^{3}}{h^{6}} a_{k, 0} k^{5} .
$$

\subsubsection{Generalization to ROS}

In this section, using the constitutive models described above, a generalized model for the randomly oriented strand case is derived.

The derivation is performed in the column representation of the ROS configuration such as the one shown in Figure 2. At a given stage, there is a minimum number of strands $p$ through thickness in the plate. The lowest column in the column representation has an index $p$. Because the deformation tends to squeeze strands, and fill the interstrand voids, at a given location, the number of strands cannot reduce. The empty columns with indices $i$ lower than $p$, for which $a_{i}=0$, will therefore stay empty. These columns are called non-deformable. Subsequently, in the column representation, a height $h_{n d}$ of the system is filled and not deformable. This height is discarded, and only the deformable part is considered in the following. 
As the ROS is being compressed, the highest column starts being squeezed. When this column reaches the height of the second highest column, both columns are squeezed together, and so on. Therefore, summing the forces $F_{k}$ applied on each of these squeezed columns results in the total reacting force applied by the press:

$$
F=\sum_{k=j(t)}^{n} F_{k}
$$

where $j(t)$ is the index of the smallest columns being currently squeezed. Note that $j(t)$ is likely to evolve during the process, and thus depends on time $t$.

Within each column, the strands orientation is random. Two strands in contact are therefore more likely not to be oriented in the same direction. Moreover, only a fraction of the strands in the column are deformed, because of the non deformable height. Some squeezed strands are therefore constrained between two non deforming strands. Based on these two remarks, a no slip condition is assumed at each interface, resulting in the case 2 in section 3.1.3. Each force $F_{k}=F_{k}^{(2)}$ then follows equation (17), such that:

$$
F=-\mu \frac{d h}{d t} \frac{w_{0}^{2} \delta^{3}}{h(t)^{6}} \sum_{k=j}^{n} a_{k, 0} k^{5}
$$

$a_{k, 0}$ being the initial area of column $k$. Industrial presses are usually controlled in closing force $F$. Therefore, the gap between the platens $h(t)$ is the unknown. It is ruled by the ordinary differential equation:

$$
\frac{d h}{d t}=-\frac{F}{\mu A} \frac{h(t)^{6}}{w_{0}^{2} \delta^{3}} \frac{1}{s}
$$

where $s(t)$ is the interstrand stacking number, that has no unit, and is defined as:

$$
s(t)=\sum_{k=j(t)}^{n} \frac{a_{k, 0}}{A} k^{5}
$$

$A$ being the total sample area.

The closing rate $d h / d t$ given in equation (20) is inversely proportional to the stacking number $s . s$, represents how the stacking configuration is limiting the flow at the strand level. Several cases can be discussed:

- If the columns currently being squeezed have a higher initial area $a_{k, 0}, s$ will be higher and the closing rate diminished.

- For a a higher number of strands $k$ in a column, $s$ is highly increased (power 5), therefore highly reducing the closing rate. This reflects that squeezing a strand twice thicker requires a lower force than squeezing a stack of two strands.

- As the number of columns being squeezed increases ( $j$ decreases), the number of terms in $s$ increases and the closing rate decreases. 
The interstrand stacking number $s(t)$ is an instantaneous number that reflects how the strands configuration will influence the compression. Note that knowing the current column configurations (given by the series of $a_{i}$ ), one can determine the columns currently compressed of indexes $k \in[j, n]$ and thus obtain the instantaneous interstrands stacking number $s(t)$.

\subsection{Implementation}

To solve the problem stated above, the gap between the platen $h(t)$, and the strand configuration given by the series $a_{i}(t)$, are to be found at each time. To this end, an unknown $X(t)$ is defined as the concatenation of $h(t)$ and the $a_{i}(t)$ :

$$
\{\boldsymbol{X}\}=\left\{\begin{array}{c}
h \\
a_{0} \\
a_{1} \\
\cdot \\
\cdot \\
\cdot \\
a_{i} \\
\cdot \\
\cdot \\
\cdot \\
a_{n}
\end{array}\right\} .
$$

Given a current vector $\boldsymbol{X}(t)$, the platen gap $h(t)$ is known and the columns currently squeezed $k \in[j, n]$ can be deduced. Moreover, the initial configuration $a_{i, 0}$ is given as a material property (equation 1). Therefore, the stacking number $s(t)$ can be computed using equation (21), subsequently giving the right hand side of equation (20).

Once the closing rate $d h / d t$ is known, the evolution rates $d a_{i} / d t$ can be inferred for each column $i$ :

- There is a minimum number of strand $p(t)$ in the lowest column, as shown on figure 2 . The columns of index lower than $p(t)$ are filled and cannot deform. For $i<p, a_{i}=0$ and $d a_{i} / d t=0$. A height $h_{n d}=\delta(p-1)$ will not be deformed.

- For $i \geq j$, the columns $a_{i}$ are simultaneously squeezed. Incompressibility of the deformed part of the columns (a height $h-h_{n d}$ ) gives:

$$
\begin{aligned}
\frac{d\left(a_{i}\left(h-h_{n d}\right)\right)}{d t} & =\frac{d a_{i}}{d t}\left(h-h_{n d}\right)+\frac{d h}{d t} \cdot a_{i} \\
& =0
\end{aligned}
$$

- To ensure conservation of mass, this matter coming from the squeezed column is to be distributed on the lower fillable columns with indices $p$ to $j$. As a first simple assumption, in the present scheme, this 
matter is considered to fill the lowest available column only. Therefore, for $i=p$, the lowest column $a_{p}$ is filled with the sum of matter coming from the above squeezed column:

$$
\frac{d a_{p}}{d t}=-\sum_{k=j}^{n} \frac{d a_{k}}{d t}
$$

- For $i>p$ and $i<j$, the columns $a_{i}$ are then assumed not to be filled, and are not squeezed either. They do not deform at all and:

$$
\frac{\partial a_{i}}{\partial t}=0
$$

This solving scheme is summarized in table 2. Concatenating the above rates $d a_{i} / d t$ in a residual vector $R$, the unknown evolution rate writes

$$
\frac{d\{\boldsymbol{X}\}}{d t}=\left\{\boldsymbol{R}\left(X, a_{i, 0}, F, \mu, \delta, w_{0}\right)\right\}
$$

Note that in order to assemble this residual vector, the indices $j(t)$ and $p(t)$, which are also functions of $X(t)$, have to be determined first.

Using the initial value of $\{\boldsymbol{X}\}$ given by the initial platen gap $h_{0}$ and the Poisson distribution equation (1), the evolution of $\{\boldsymbol{X}\}$ is obtained versus time by solving the ordinary differential equation (26). To proceed, a fourth order Runge Kutta numerical method is used in MATLAB (ode 45 function). The MATLAB code is available at https://github.com/arthurlevy/ISVC/.

\section{Experimental methods}

\subsection{Material}

The material used in this study is a Carbon / PEEK (PolyEtherEtherKetone) bulk moulding compound composite. It consists of strands of unidirectional prepreg tape cut to desired length. The fibre volume fraction is about $60 \%$.

The bulk moulding compound is available in different width and length, but its thickness $\delta$ is fixed for a preimpregnation line. The thickness of the strand was measured by manufacturing a 32 plies quasi-iso laminates using the identical prepreg tape in continuous form. The plate thickness was measured to be $4.34 \pm 1 \% \mathrm{~mm}$ at room temperature. This comes down to a ply thickness

$$
\delta=0.136 \mathrm{~mm}
$$

at room temperature.

The density $\rho$ of the material was measured in water using the ASTM D792 standard [24]. At room temperature,

$$
\rho=1560 \mathrm{~kg} \cdot \mathrm{m}^{-3} \text {. }
$$




\subsection{Instrumented hot press}

The manufacturer's recommended consolidation temperature is between 370 and $400{ }^{\circ} \mathrm{C}$. To measure the compaction behaviour at such a temperature, a specific instrumented hot press was designed (figure 7a). It consists of heated platens in a die set that ensure parallelism.

The platens are heated using four $500 \mathrm{~W}$ electric cartridges. A controlling type $\mathrm{K}$ thermocouple is located in the middle of each platen, $2 \mathrm{~mm}$ underneath the surface. The temperature is controlled with two independent SD Series PID controller from Watlow.

The H13 steel platens are polished to ensure an easy removal. A fitting polished steel frame is positioned around the lower platen which has an area

$$
A=4^{\prime \prime} \times 4^{\prime \prime}=101.6 \mathrm{~mm} \times 101.6 \mathrm{~mm} \text {. }
$$

The frame ensures that the material is not squeezed out of the gap when pressure is applied. The upper platen has an area of $3.99^{\prime \prime} \times 3.99^{\prime \prime}=101.3 \mathrm{~mm} \times 101.3 \mathrm{~mm}$, and fits inside the frame leaving a sufficient shear edge gap to prevent contact and friction.

The setup is positioned in a $250 \mathrm{kN}$ MTS tensile machine that controls of the applied force and measures the closing of the mould $h(t)$.

\subsection{Procedure}

The tests are performed at $380^{\circ} \mathrm{C}$. At this temperature the equivalent Newtonian viscosity of the composite was measured in a previous work [25]:

$$
\mu=4.0110^{4} \mathrm{~Pa} . \mathrm{s}
$$

First, the mould is coated with Freekote 700-NC release agent. A given amount of strands is weighted using a precision scale. It is then manually positioned randomly in the mould. The strands are positioned as much as possible homogeneously over the whole mould cavity. This minimizes the macroscopic flow of strands and ensures mostly an ISVC reduction deformation mechanism.

The upper platen is then moved down until a contacting force is measured. Note that this low contacting force is only linked to the elastic packing deformation, and is considered to induce a negligible flow during the subsequent heating phase. Heating is then started and temperature reaches $380^{\circ} \mathrm{C}$ in about $15 \mathrm{~min}$. An additional stabilization time of $10 \mathrm{~min}$ ensures isothermal condition in the material during processing.

The closing force is then applied in a one second ramp and maintained thereafter. A dwell time of 5 min is allowed before cooling down.

Cooling is performed using compressed air that is pulsed into designed canals in both platens. The cooling rate is not controlled but is about $-15^{\circ} \mathrm{Cmin}^{-1}$. When temperature drops below $143^{\circ} \mathrm{C}$, which is the glass transition temperature of PEEK [26], the mould is opened and the plate is ejected. 
Time, force and displacement signals are acquired during the whole test, at a rate of $4 \mathrm{~Hz}$.

The final thickness $h_{f}$ of the compression moulded plate is measured using a micrometer.

\subsection{Thickness correction}

Because of the thermal dilatation occurring during the cooling, corrections have to be performed using the transverse integral thermal expansion $\varepsilon_{t h}$ of the material between room temperature and processing temperature $\left(380^{\circ} \mathrm{C}\right)$. The thickness of the specimen at processing temperature writes:

$$
h_{f}^{380}=h_{f}\left(1+\varepsilon_{t h}\right) .
$$

$\varepsilon_{t h}$ is measured with a TA instruments Q400 thermomechanical analyzer on a quasi-isotropic laminate, and is supposed to be similar for the randomly oriented strand specimens (mostly transverse behaviour):

$$
\varepsilon_{t h}=7.39 \%
$$

The specimen final thickness at processing temperature $h_{f}^{380}$ is useful to offset the acquired relative displacement measurement and obtain absolute thickness data.

Similarly, the ply thickness $\delta$ at processing temperature is found to be

$$
\delta^{380}=0.146 \mathrm{~mm}
$$

All the thickness mentioned hereunder are that at processing temperature.

\section{Validation}

\subsection{Test matrix}

Tests A, B, C and D were performed using the process and material parameters given in table 3. Case $\mathrm{A}$ is the baseline, case $\mathrm{B}$ is manufactured with larger strands, case $\mathrm{C}$ with a higher closing force, and case $\mathrm{D}$ with a reduced amount of material (i.e. a thinner part).

\subsection{Experimental results}

The platen gap versus time is plotted for the four cases in Figure 8. Starting from a thick initial gap (the bulk factor is above 200\%), a fast compaction initially occurs. This points out that the final ISVC did not reach 0 , and the plates are not fully consolidated. As given in Table 3, case $\mathrm{C}$ shows a final thickness slightly smaller than the expected nominal thickness $h_{\infty}^{380}$. This might be explained, for this high pressure test, by the presence of flashes at the shear edge location. The amount of material in the final plate was thus reduced.

For the four case, the thickness reaches an asymptotic value after no more than 3 minutes. But the expected final nominal thickness $h_{\infty}^{380}$, shown in dotted lines on the graph, is not reached for cases A, B and D. For these cases, the processing conditions will not be sufficient to ensure full consolidation, even for longer dwell times. 


\subsection{Model validation}

The thickness predictions using the presented model are plotted on the same graph (Figure 8). The model is accurate at determining the final asymptotic plateau. As can be noticed on table 3 , the error on the final thickness (at time $t=5$ min which corresponds to the dwell duration) never exceeds $5 \%$, with a coefficient of variation of $3 \%$ between model and experiment.

This small error on the thickness prediction is enhanced when considering the ISVC percentage. Table 3 shows that the absolute error reaches $5 \%$ for case B. Nonetheless, for the four case, the trend in terms of ISVC is recovered.

Figure 8 also shows a discrepancy at the beginning of the compaction. An explanation for this discrepancy is likely the misevaluation of the initial condition for the ODE equation 26. The initial vectors $a_{i}$ of the column representation where considered to follow the ideal Poisson distribution. But as suggested above (section 2.3), the initial condition might differ for real random initial placement of strands.

Improvements of the model could be performed in order to evaluate better this initial condition. Nonetheless, in it current form, the model only uses physical and geometrical parameters. Moreover, almost all these parameters in the model are straightforward to obtain. The only nontrivial parameter is the equivalent transverse viscosity, that was characterized in a previous work [25], using a squeeze flow setup. For a different material or temperature system, this single missing parameter could also be identified using a single parameter basic inverse method. Finally the model is kept as is in the present paper.

\section{Application}

\subsection{Parametric study}

In this section the model is used to investigate the effect of various process parameter on the interstrand void reduction. Case A is used as a baseline.

\subsubsection{Effect of initial strand geometry}

This section discusses the effect of the width and length of the strands on the compaction. Because the thickness of the prepreg material is given by the manufacturer, and is difficult to modify, its influence is not addressed hereunder.

As shown on equation (20), the closing rate is inversely proportional to the square of the strand initial width $w_{0}$. The narrower the strands, the faster the compaction. This is confirmed by the simulations that predicted a final ISVC of $2.2 \%, 4.5 \%$, and $9.2 \%$ after 5 min for respective increasing strand widths of $3.18 \mathrm{~mm}$ (baseline, case A), $6.35 \mathrm{~mm}$, and $12.70 \mathrm{~mm}$.

The model also shows that the closing rate does not depend on the initial strand length $L_{0}$. Nonetheless, for long strands, the packing stress phenomena that is associated with the bending of the strands might become non negligible. Moreover, besides the ISVC reduction, the strand length $L_{0}$ is likely to influence the macroscopic squeeze flow. 


\subsubsection{Effect of part thickness}

Case $\mathrm{D}$ is the compression of a thinner plate. This corresponds to a different number of strands $N_{s}$ in the plate. Note that in this case, The model is the same except that:

1. The initial thickness $h(t=0)$ is reduced.

2. The initial distribution, given by equation (1), is spread over a smaller range of indexes since $\tilde{n}$ is reduced.

The predicted ISVC at early time $t=1 \mathrm{~min}$ increases from $5.0 \%$ to $6.14 \%$ when the plate thickness decreases from $2.67 \mathrm{~mm}$ (baseline, case A) to $1.67 \mathrm{~mm}$. Increasing the part thickness improves the interstrand void reduction.

\subsubsection{Effect of strands distribution}

The initial distribution of strand was considered to follow the ideal Poisson distribution equation (1). Nonetheless, section 2.3, showed that a particular realization of the initial configuration $a_{i, 0}$ might deviate from this ideal distribution. Especially for small number of strands. To investigate this deviation, for the three cases described in table 1 , fifty realizations of the initial distribution $a_{i, 0}$ were generated using the algorithm proposed by Knuth [18]. The model was then applied using these initial conditions, and fifty final ISVC were predicted for each case. The variability of the final ISVC is reported in term of standard deviation on table 1.

These results show that the smaller the number of strand the higher the variability in the final ISVC. In the case of a non-homogeneous material with very few strands (the Extreme case), the variability in the predicted final void content reached $10 \%$. Nonetheless, in the industrial case, the standard deviation drops below $2 \%$ of the mean final value. Thus, for these kind of industrial cases involving a large number of strands, the use of the ideal Poisson distribution and the deterministic presented model is valid.

\subsubsection{Effect of process parameters}

In equation (20) and (21) the closing speed is inversely proportional to the characteristic time

$$
\tau=\frac{\mu A}{F} .
$$

The closing speed appears linearly dependent on applied closing pressure $P=F / A$. It is also inversely proportional to the melt viscosity.

The viscosity dependency is highly dependent on the temperature. An Arrhenius law [27, 28]:

$$
\mu(T)=A \exp \left(\frac{E_{a}}{R T}\right)
$$

can express the temperature dependency of the viscosity. Increasing the processing temperature from $380^{\circ} \mathrm{C}$ to $400{ }^{\circ} \mathrm{C}$ will result in an increase of $12.6 \%$ of the closing speed ${ }^{1}$.

\footnotetext{
${ }^{1}$ Using $E_{a} / R=2969 \mathrm{~K}$ as suggested by Sonmez and Hahn [27].
} 


\subsection{Design guidelines}

Considering a given strand thickness $\delta=0.146 \mathrm{~mm}$ at $380^{\circ} \mathrm{C}$, a given processing temperature (and thus a given viscosity $\mu$ ), and an ideal Poisson distribution for the initial configuration, equation (20) shows that the model only depends on two key parameters:

- the closing pressure to squared strands width ratio $P / w_{0}^{2}$

- the nominal thickness $h_{\infty}$, or seamlessly the amount of strands in the mould, that rules the initial thickness $h(t=0)$ and initial column distribution.

The model was solved for a wide variety of these two parameters, for a 5 min dwell time at $380^{\circ} \mathrm{C}$ and thus a constant viscosity $\mu=4.0110^{6} \mathrm{~Pa}$.s. The iso-values of the final ISVC are plotted on the design graph shown on the top of figure 9 . The horizontal axes being the ratio $P / w_{0}^{2}$, and the vertical one, the nominal final thickness $h_{\infty}$ at room temperature.

Because the parameter $P / w_{0}^{2}$ is not trivial to estimate, the lower part of the design chart was added. It allows for a fast evaluation, for available strand width of 1/16, 1/8, $1 / 4$ and $1 / 2$ in (respectively 1.59, 3.18, 6.35 and $12.7 \mathrm{~mm}$ ). The use of the graph for case A detailed above, is shown with arrows on the chart. First the value $P / w_{0}^{2}$ is obtained with the lower graph for a closing pressure of 4.8 bar and a strand width of $1 / 8$ in $(3.18 \mathrm{~mm})$. Then, in the case of a final nominal thickness $h_{\infty}=2.48 \mathrm{~mm}$, the upper graph gives the final ISVC of

$$
\phi=2.2 \%
$$

which is in agreement with the value presented above (table 3 ).

This chart can be used to easily determine the processing window. It provides useful guidelines to the process designer. Nonetheless, in the case of different processing temperature (different viscosity) or different dwell times the map should be reconstructed.

\section{Conclusion}

Press forming of randomly oriented composite strands can process complex parts fast, with final properties better than short fibres reinforced polymer injected moulded parts. During this forming process several deformation phenomena occur.

In this paper, a modelling of the interstrand void content reduction due to the spreading of each strand was proposed. A column representation was adopted to efficiently handle the random configuration of a very large number of strands in a mould. Then, starting from the squeeze flow behaviour of one single strand, a constitutive model was elaborated to predict the compression and thus the interstrand void reduction. The model accounts for the closing pressure, the equivalent viscosity of the material, geometry of the strands, and the initial distribution of strands via the column representation. 
Experimental validation was performed using a specially designed instrumented hot press. The press sits in a MTS tensile machine that measures the platen gap evolution while controlling the closing force. The model was validated on four different configurations where the final part thickness was accurately predicted.

A parametric study was performed using the present model. The effects of closing force, geometry of the strands, temperature and time were investigated. Finally, a design chart was constructed. It visually gives the final interstrand void content for any strand width, part thickness and closing pressure. The design chart can readily be used to determine processing windows that ensure a final interstrand void content below a given objective.

\section{Acknowledgements}

This study was made possible through the financial support provided by the industrial and institutional partners: Bell Helicopter Textron Canada Limited, Bombardier Inc., Pratt and Whitney Canada Corp., Marquez Transtech Limited, Delastek Inc., Avior Integrated Products Inc., the Natural Sciences and Engineering Research Council (NSERC) and the Consortium for Research and Innovation in Aerospace in Quebec (CRIAQ).

The authors would like to thank Gilles Philippe Picher Martel, Benoit Landry and Dominic LeBlanc from McGill, and Ali Yousefpour and Steven Roy from the National Research Council of Canada Aerospace Manufacturing Technology Centre, for the instrumented hot press design, manufacturing and setup. Benoit Landry also performed the TMA measurements at McGill.

\section{References}

[1] Sepehr M, Carreau PJ, Moan M, Ausias G. Rheological properties of short fiber model suspensions. Journal of Rheology 2004;48(5):1023-49.

[2] Le Corre S, Dumont P, Orgéas L, Favier D. Rheology of highly concentrated planar fiber suspensions. Journal of Rheology $2005 ; 49(5): 1029-58$.

[3] Feraboli P, Peitso E, Deleo F, Cleveland T, Stickler PB. Characterization of Prepreg-Based Discontinuous Carbon Fiber/Epoxy Systems. Journal of Reinforced Plastics and Composites 2008;28(10):1191-214.

[4] Wijngaarden JV, Jongbloed A, de Vries J. Thermoplastic Compound Compression Molding. In: Sampe. Seattle, WA, USA; 2010,

[5] Selezneva M, Picher-Martel GP, Landry B, Lessard L, Hubert P, Trudel-Boucher D, et al. Compression Moulding of Discontinuous-fibre carbon/PEEK Composites: Study of Mechanical Properties. In: SAMPE Conference - Baltimore. 2012,.

[6] Picher-Martel GP, Levy A, Hubert P. Squeeze Flow of Randomly-Oriented Strands Thermoplastic Composites: Tracing the Flow Front. In: ICCM 19. Montreal, Canada; 2013,.

[7] Eguémann N, Giger L, Roux M, Dransfeld C, F. T, D. P. Compression Moulding of Complex Parts for the Aerospace With Discontinuous Novel And Recycled Thermoplastic Composite Materials. In: 19th International Conference on Composite Materials. Montreal, Canada; 2013, p. 6616-26.

[8] Luchoo R, Harper LT, Bond MD, Dodworth A, Warrior NA. Macro-scale Modelling of Discontinuous Fibre Composites. In: 18th International Conference on Composite Materials; vol. 1. 2011,.

[9] Servais C, Michaud V, Månson JA. The Packing Stress of Impregnated Fiber Mats. Polymer Composites 2001;22(2):298311.

[10] Servais C, Luciani A, Månson JA. Fiber-fiber interaction in concentrated suspensions: Dispersed fiber bundles. Journal of rheology 1999;43(4):1005-18.

[11] Leterrier Y, G'Sell C. Formation and elimination of voids during the processing of thermoplastic of matrix composites. Polymer Composites 1994;15(2):101-5.

[12] Varna J, Joffe R, Berglund LA, Lundstriim TS. Effect of Voids on Failure Mechanisms in RTM Laminates. Composites Science and Technology 1995;53:241-9. 
[13] Ranganathan S, Advani SG, Lamontia MA. A Non-Isothermal Process Model for Consolidation and Void Reduction during In-Situ Tow Placement of Thermoplastic Composites. Journal of Composite Materials 1995;29(8):1040-62.

[14] Khan MA, Mitschang P, Schledjewski R. Tracing the void content development and identification of its effecting parameters during in situ consolidation of thermoplastic tape material. Polymers and Polymer Composites 2010;18(1):1-15.

[15] Dai C, Steiner PR. Compression behavior of randomly formed wood flake mats. Wood and fiber science 1993;28(1):45-51.

[16] Hall P. Introduction to the theory of coverage processes. New York: Wiley; 1988.

[17] Dai C, Steiner PR. Spatial structure of wood composites in relation to processing and performance characteristics. Part 2. Modelling and simulation of a randomly-formed flake layer network. Wood Science and Technology 1994;28(2):135-46.

[18] Knuth DE. The Art of Computer Programming - Seminumerical Algorithm. third ed.; Reading, MA: Addison-Wesley; 1997.

[19] Wang E, Gutowski T. Laps and gaps in thermoplastic composites processing. Composites Manufacturing 1991;2(2):69-78.

[20] Shuler SF, Advani SG. Transverse squeeze flow of concentrated aligned fibers in viscous fluids. Journal of Non-Newtonian Fluid Mechanics 1996;65:47-74

[21] Gagné Brulotte X. Aspects of In-situ Consolidation of Thermoplastic Laminates Manufactured by Automated Tape Placement: A Material Deformation Study. Ph.D. thesis; McGill University; 2012.

[22] Picher-Martel GP, Levy A, Hubert P. A Comparative Study of the Squeeze Flow Behaviour of Unidirectional and Randomly-Oriented Strands Carbon/PEEK Composites during Compression Moulding. Composites Part A: Applied Science and Manufacturing 2014; under revi.

[23] Laun H, Rady M, Hassager O. Analytical solutions for squeeze flow with partial wall slip. Journal of Non-Newtonian Fluid Mechanics 1999;81(1-2):1-15.

[24] ASTM International . ASTM D792 - 00. Standard Test Method for Density and Specific Gravity (Relative Density) of Plastics by Displacement. 2000.

[25] Picher-Martel GP, Hubert P. Squeeze flow behavior of Carbon/PEEK randomly oriented strands under transverse compaction. In: American Society for Composites 27th Annual Technical Conference. 2012,

[26] Cogswell FN. Thermoplastic aromatic polymer composites. Cambridge UK.: Woodhead; 1992.

[27] Sonmez F, Hahn H. Analysis of the on-line consolidation process in thermoplastic composite tape placement. Journal of Thermoplastic Composite Materials 1997;10(6):543-72.

[28] Levy A, Le Corre S, Poitou A. Ultrasonic welding of thermoplastic composites: a numerical analysis at the mesoscopic scale relating processing parameters, flow of polymer, and quality of adhesion. International Journal of Material Forming 2014;7(1):39-51. 


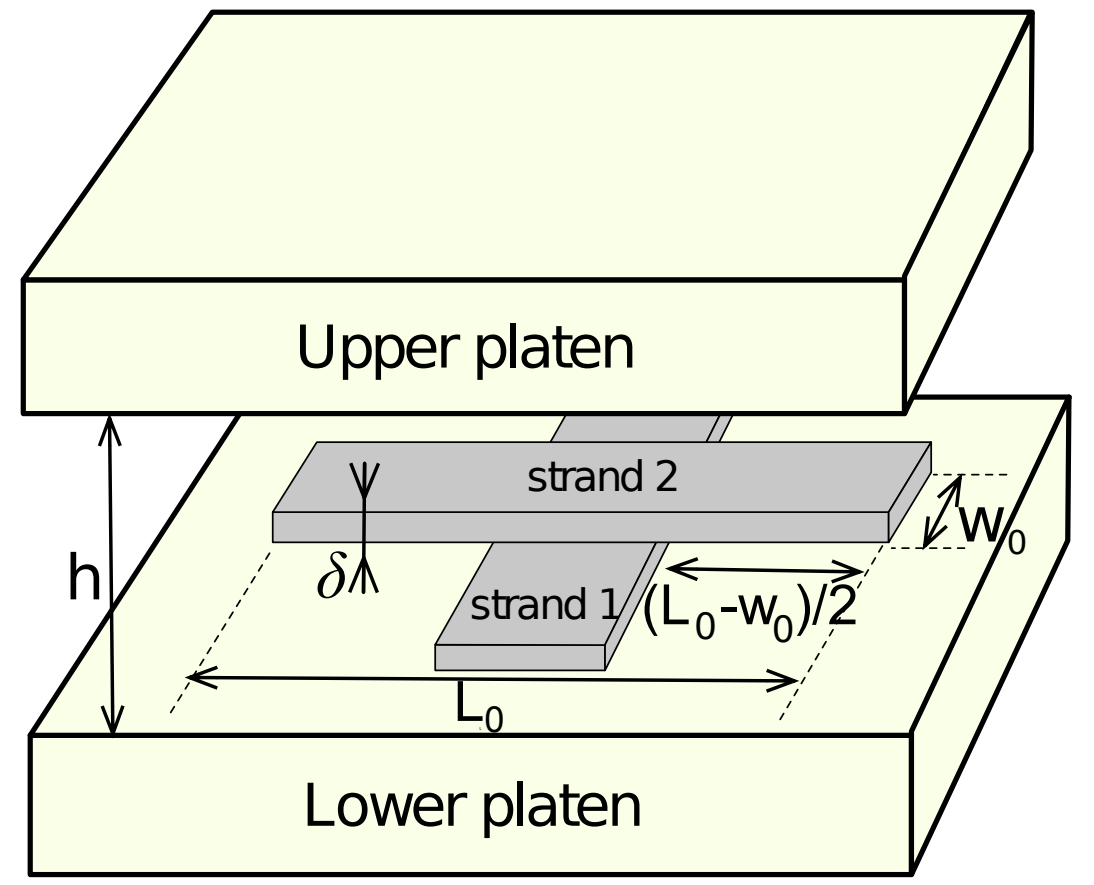

(a) Real configuration

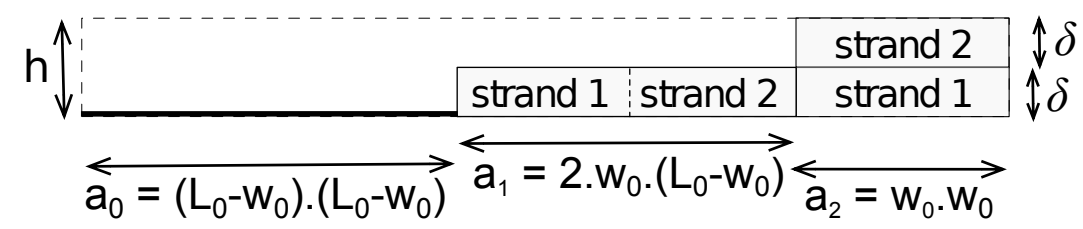

(b) Column representation.

Figure 1: Basic stacking of two strands. The real geometrical configuration corresponds, in the column representation, to a series of three different columns. 


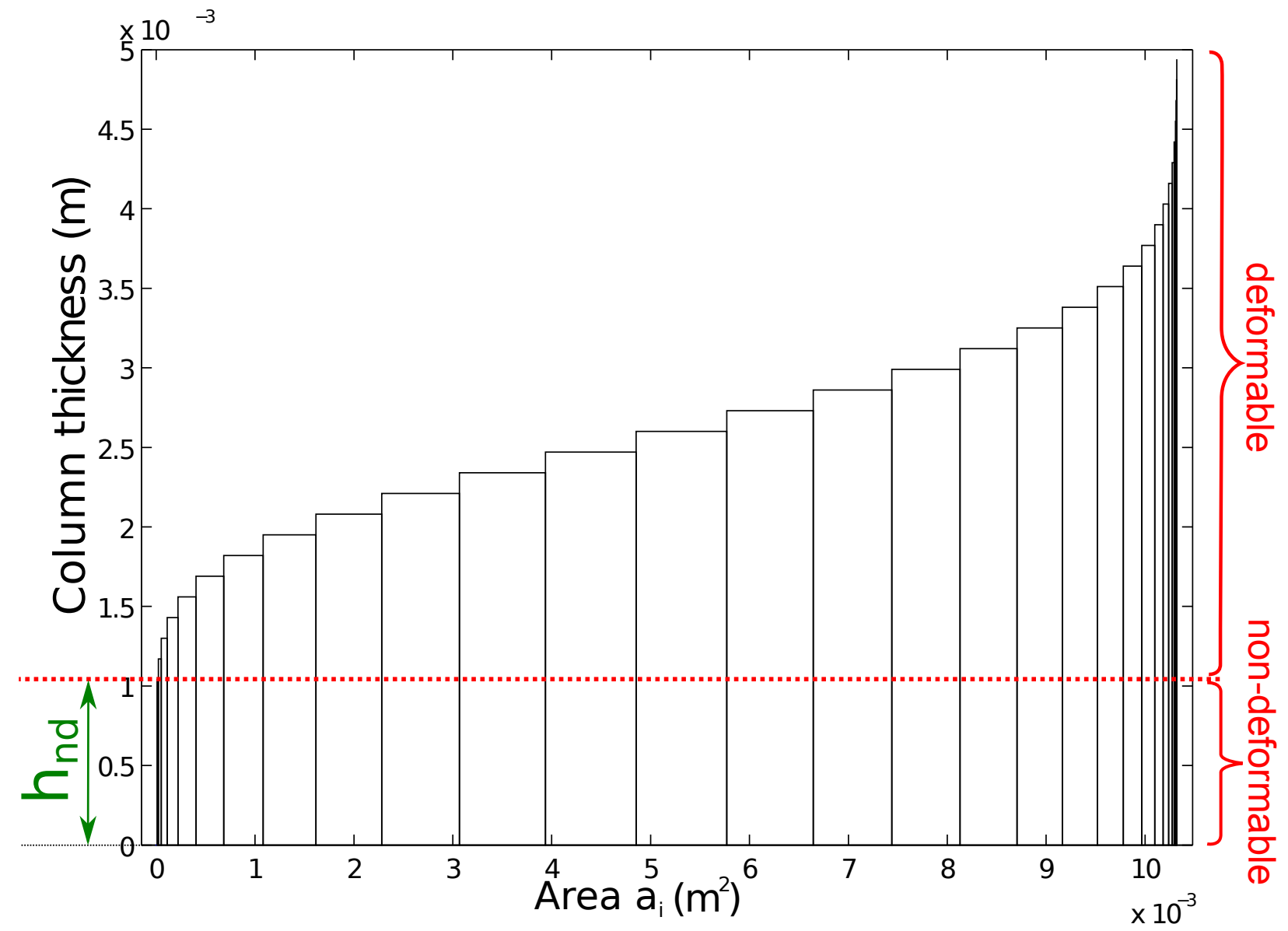

Figure 2: Column representation of randomly oriented strands $(\tilde{n}=20)$. A part of the space is filled and therefore nondeformable. 


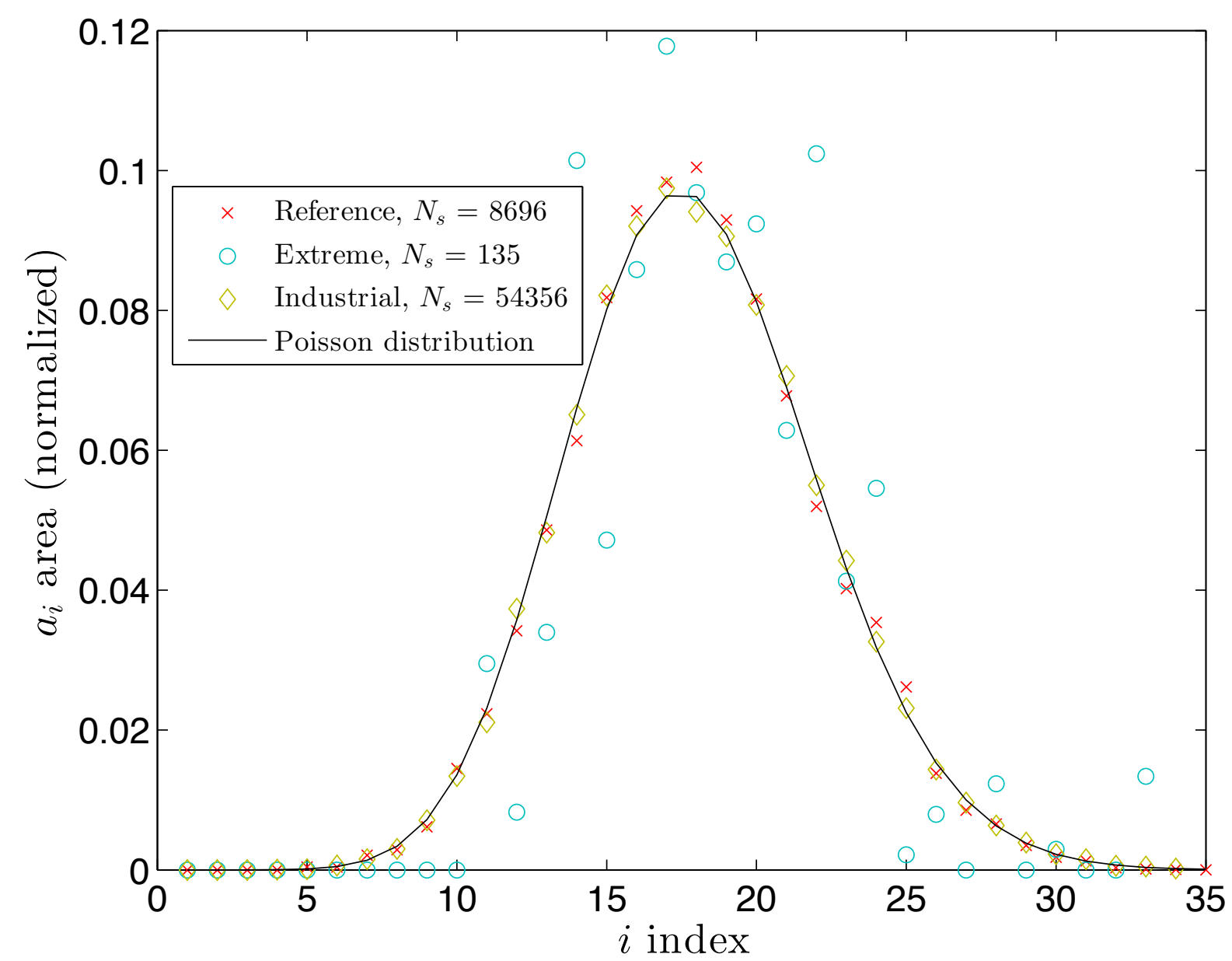

Figure 3: Three realizations of initial $a_{i}$ distribution for different number $N_{s}$ of strands in the mould as detailed in table 1 . The ideal curve, that would be reached for an infinite number of strand, is the Poisson distribution equation (1). 


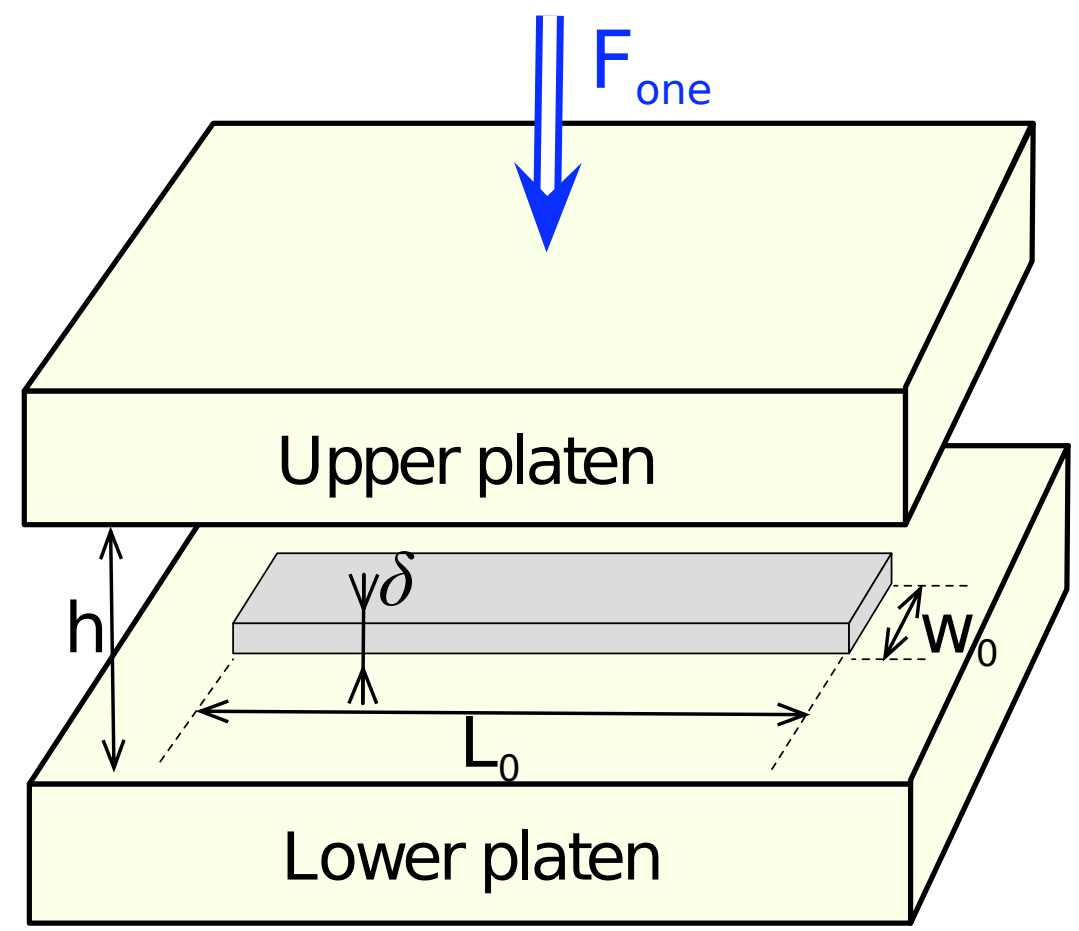

(a) Real geometry.

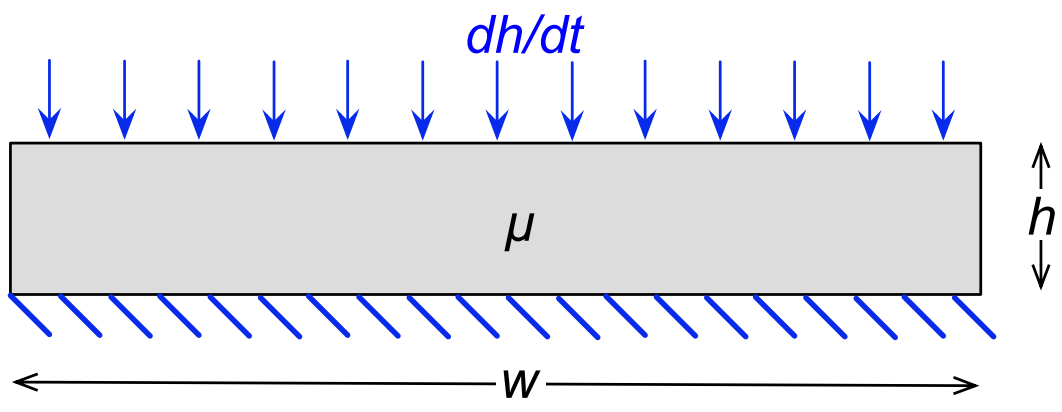

(b) Two-dimensional modelling using plain strain assumption.

Figure 4: Compression of a single strand. 


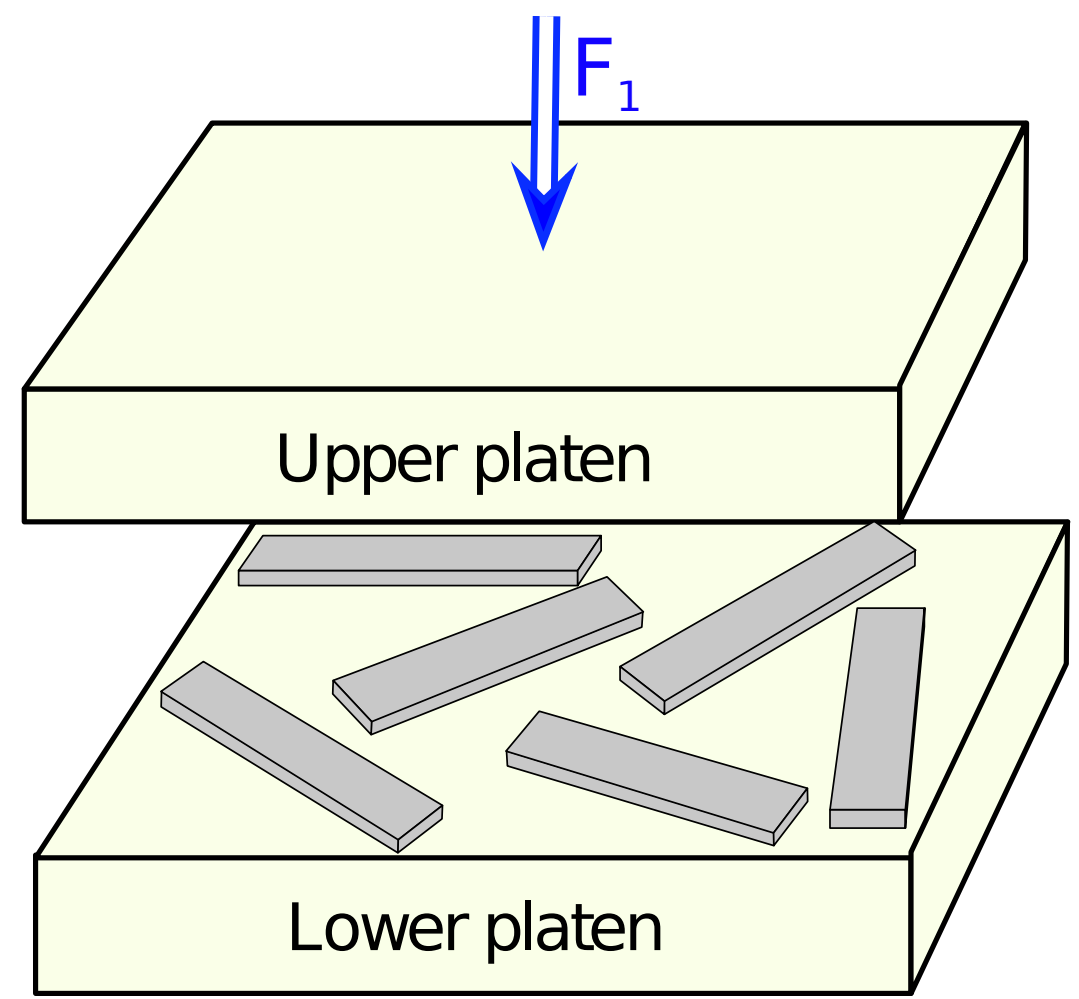

(a) Example of a random repartition of non overlapping strands.

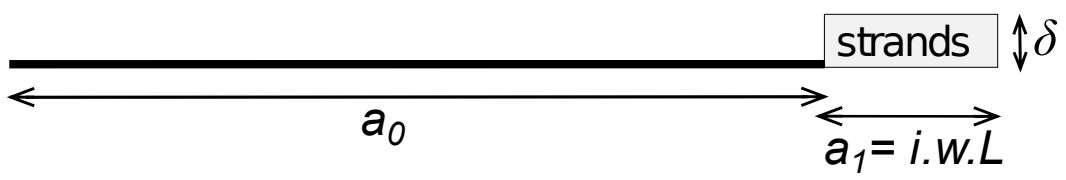

(b) Equivalent column representation: one empty column and one column of area $a_{1}$ and height $\delta$

Figure 5: Squeezing of several non overlapping strands. 


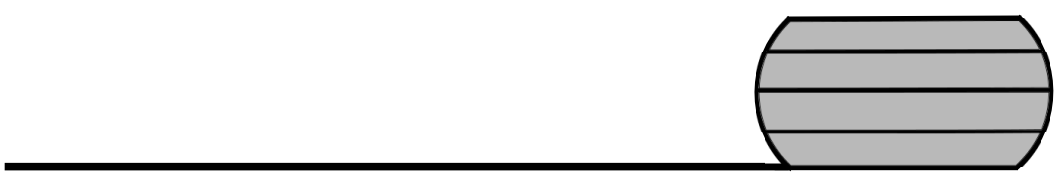

(a) Case 1. The stacking behaves as one single thick strand.

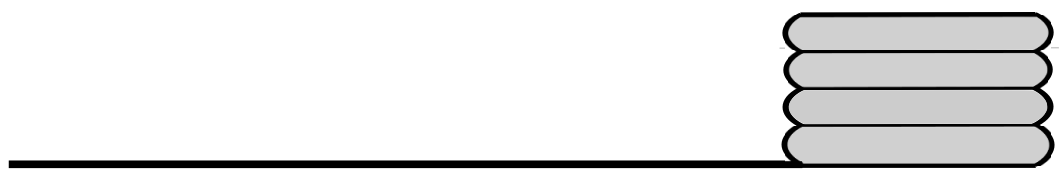

(b) Case 2. Each single strand deform independently.

Figure 6: Stacking of strands and its two extreme deformation modes in the column representation. 


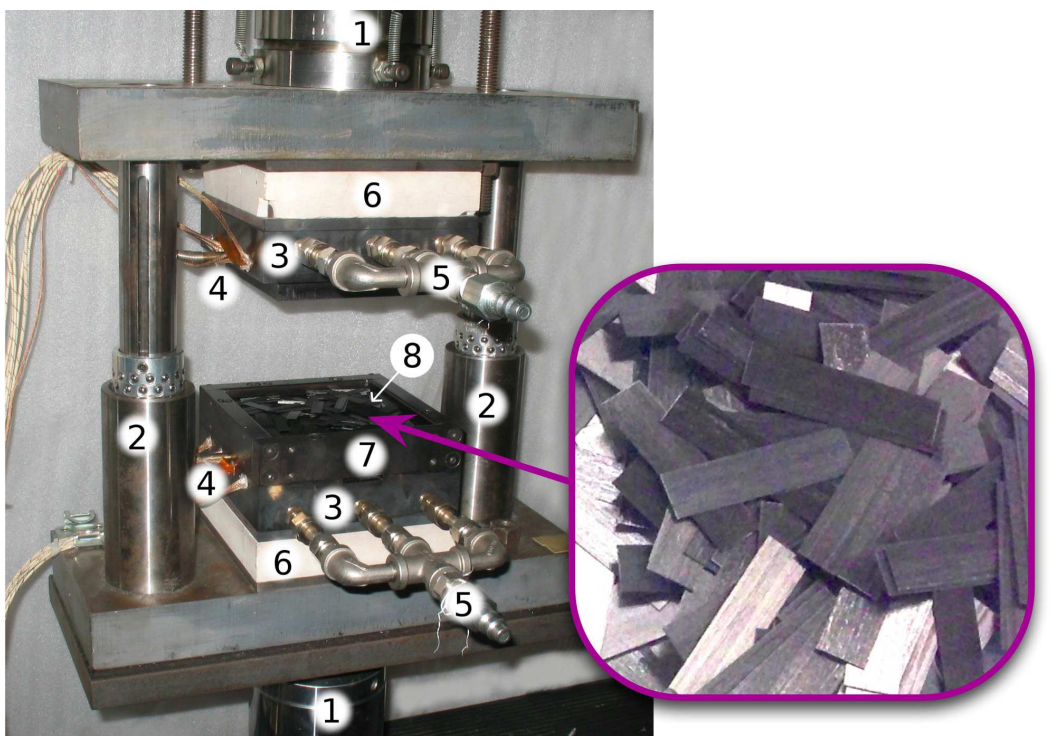

(a) Instrumented hot press. 1: attachment to the tensile machine. 2: ball bearing die set. 3: heated platens. 4: heating cartridges and thermocouple. 5: air cooling system. 6: thermal insulation. 7: frame. 8 and close-up: ROS material ready to be processed.

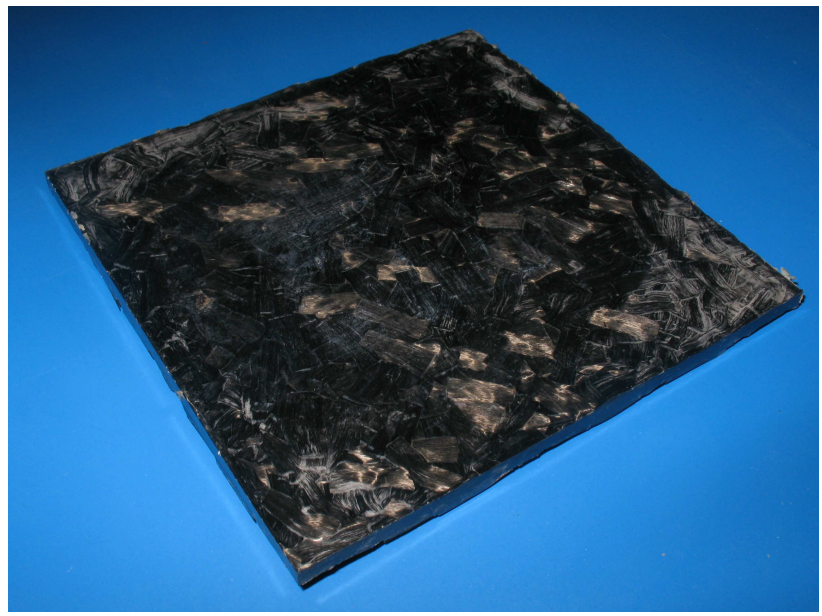

(b) Net shape flat plate obtained with this mould.

Figure 7: Experimental manufacturing of Randomly Oriented Strands (ROS). 


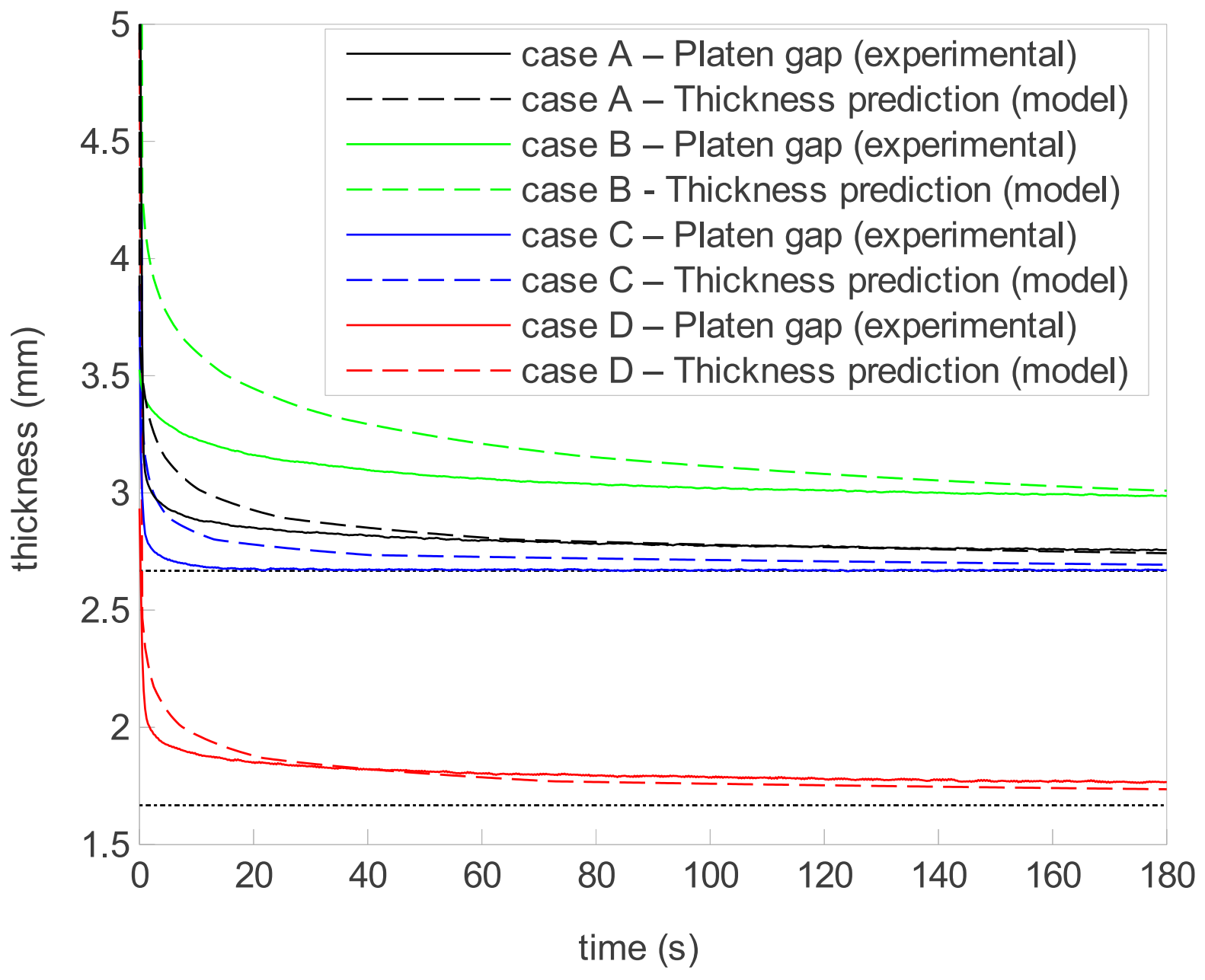

Figure 8: Experimental and modelled platen gaps versus time. The four cases conditions are detailed in table 3. 


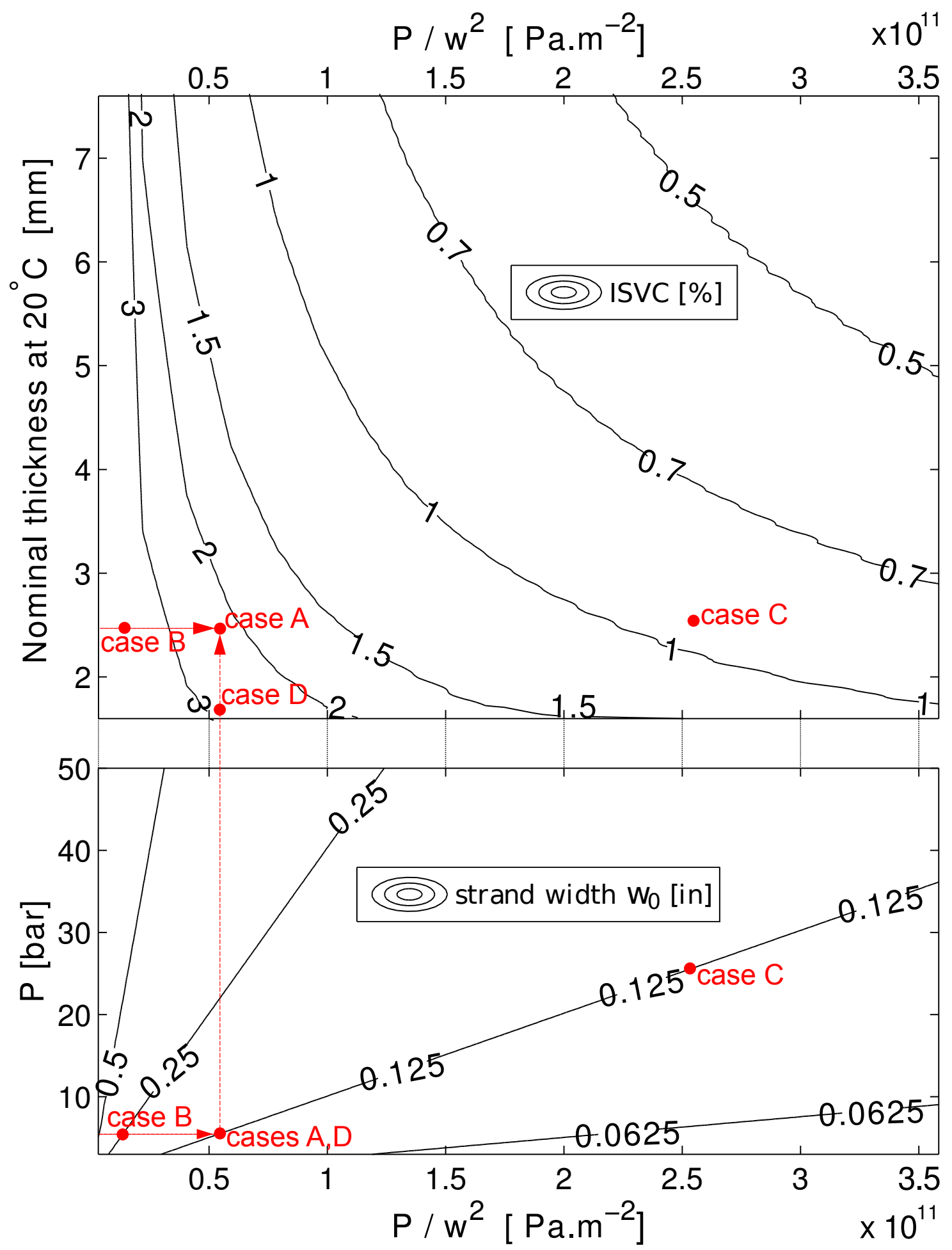

Figure 9: Design chart giving the interstrand void content after 5 min dwell at $380^{\circ} \mathrm{C}$, for any closing pressure $P$, initial strand width $w_{0}$, and expected nominal part thickness $h_{\infty}$ at room temperature. The red arrows show how to use the graph for the specific case A. 
Table 1: Initial strand distribution analysis: the three cases studied.

\begin{tabular}{llccc}
\hline & & Reference & Extreme & Industrial \\
\hline Strand width & $w_{0}$ & $3.18 \mathrm{~mm}$ & $12.7 \mathrm{~mm}$ & $6.35 \mathrm{~mm}$ \\
Strand length & $L_{0}$ & $6.35 \mathrm{~mm}$ & $25.4 \mathrm{~mm}$ & $12.7 \mathrm{~mm}$ \\
Nominal plate thickness & $h_{\infty}$ & $2.48 \mathrm{~mm}$ & $2.48 \mathrm{~mm}$ & $3 \mathrm{~mm}$ \\
Mould area & $A$ & $101.6 \mathrm{~mm} \times 101.6 \mathrm{~mm}$ & $50.8 \mathrm{~mm} \times 50.8 \mathrm{~mm}$ & $508 \mathrm{~mm} \times 508 \mathrm{~mm}$ \\
Number of strands & $N_{s}$ & 8696 & 135 & 54356 \\
\hline Final ISVC mean value & $\phi$ & $2.13 \%$ & $9.6 \%$ & $4.6 \%$ \\
Final ISVC standard deviation & & $0.08 \%$ & $1.5 \%$ & $0.07 \%$ \\
\hline
\end{tabular}


Table 2: Construction of the right hand side of equation (26).

\begin{tabular}{cccc}
\hline$i$ & $a_{i}$ & Status & $\frac{\partial a_{i}}{\partial t}$ \\
\hline 0 & 0 & empty, non deformable & 0 \\
$\ldots$ & 0 & empty, non deformable & 0 \\
$p-1$ & 0 & empty, non deformable & 0 \\
$p$ & $>0$ & deformable, filled & $-\sum_{k=j}^{n} \frac{\partial a_{k}}{\partial t}$ \\
$p+1$ & $>0$ & deformable, unchanged & 0 \\
$\ldots$ & $>0$ & deformable, unchanged & 0 \\
$j-1$ & $>0$ & deformable, unchanged & 0 \\
$j$ & $>0$ & deformable, squeezed & $-\dot{h} . a_{i} /\left(h-h_{n d}\right)$ \\
$\ldots$ & $>0$ & deformable, squeezed & $-\dot{h} \cdot a_{i} /\left(h-h_{n d}\right)$ \\
$n$ & $>0$ & deformable, squeezed & $-\dot{h} \cdot a_{i} /\left(h-h_{n d}\right)$ \\
\hline
\end{tabular}


Table 3: Process parameters used in the four tests.

\begin{tabular}{lccccc}
\hline & & Case A & Case B & Case C & Case D \\
\hline Amount of strands used & & $40 \mathrm{~g}$ & $40 \mathrm{~g}$ & $40 \mathrm{~g}$ & $\mathbf{2 5} \mathbf{g}$ \\
Nominal thickness at $20^{\circ} \mathrm{C}$ & $h_{\infty}$ & $2.48 \mathrm{~mm}$ & $2.48 \mathrm{~mm}$ & $2.48 \mathrm{~mm}$ & $\mathbf{1 . 5 5} \mathbf{~ m m}$ \\
Nominal thickness at $380^{\circ} \mathrm{C}$ & $h_{\infty}^{380}$ & $2.67 \mathrm{~mm}$ & $2.67 \mathrm{~mm}$ & $2.67 \mathrm{~mm}$ & $\mathbf{1 . 6 7} \mathbf{~ m m}$ \\
\hline Closing force & $F$ & $5 \mathrm{kN}$ & $5 \mathrm{kN}$ & $\mathbf{2 5} \mathbf{~ N N}$ & $5 \mathrm{kN}$ \\
Closing pressure & $P=\frac{F}{A}$ & $4.84 \mathrm{bar}$ & $4.84 \mathrm{bar}$ & $\mathbf{2 4 . 2} \mathbf{b a r}$ & $4.84 \mathrm{bar}$ \\
\hline Strands initial width & $w_{0}$ & $3.18 \mathrm{~mm}$ & $\mathbf{6 . 3 5} \mathbf{~ m m}$ & $3.18 \mathrm{~mm}$ & $3.18 \mathrm{~mm}$ \\
Strands initial length & $L_{0}$ & $6.35 \mathrm{~mm}$ & $\mathbf{2 5 . 4} \mathbf{~ m m}$ & $6.35 \mathrm{~mm}$ & $6.35 \mathrm{~mm}$ \\
\hline Final experimental thickness & $h_{f}^{380}$ & $2.75 \mathrm{~mm}$ & $2.97 \mathrm{~mm}$ & $2.66 \mathrm{~mm}$ & $1.76 \mathrm{~mm}$ \\
Final predicted thickness & $h(5 \mathrm{~min})$ & $2.73 \mathrm{~mm}$ & $2.80 \mathrm{~mm}$ & $2.69 \mathrm{~mm}$ & $1.71 \mathrm{~mm}$ \\
\hline Final experimental ISVC & & $2.9 \%$ & $10 \%$ & $0 \%$ & $4.9 \%$ \\
Final predicted ISVC & $\phi$ & $2.2 \%$ & $4.6 \%$ & $0.8 \%$ & $2.6 \%$ \\
\hline
\end{tabular}

